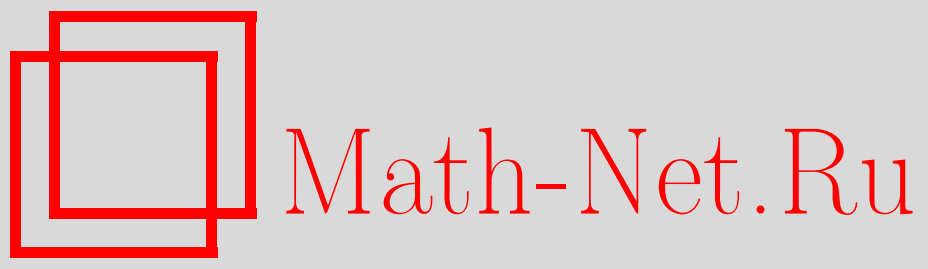

Б. А. Пламеневский, А. С. Порецкий, О. В. Сарафанов, О вычислении волноводной матрицы рассеяния для системы Максвелла, Функи. анализ и его прил., 2015, том 49, выпуск 1, 93-96

DOI: https://doi.org/10.4213/faa3175

Использование Общероссийского математического портала MathNet.Ru подразумевает, что вы прочитали и согласны с пользовательским соглашением

http://www. mathnet.ru/rus/agreement

Параметры загрузки:

IP : 54.164 .48 .24

26 апреля 2023 г., 09:32:16

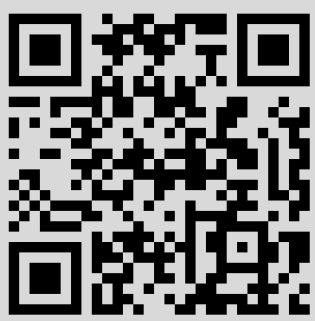


Monographs in Math., Springer-Verlag, Heidelberg-Dordrecht-London-New York, 2012. [16] И. М. Стейн, Сингулярные интегралы и дифференциальные свойства функций, Мир, М., 1973.

Санкт-Петербургский государственный университет, Лаборатория им. П. Л. Чебышева

Поступила в редакцию e-mail: juliavmeshke@yandex.ru 7 февраля 2014 г.

Санкт-Петербургский государственный университет, физический факультет

e-mail: suslina@list.ru

УДК 517.958+621.372.8

\section{О вычислении волноводной матрицы рассеяния для системы Максвелла*}

(c) 2015. Б. А. ПЛАмЕНЕВСКИЙ, А. С. ПОРЕЦКИЙ, О. В. САРАФАНОВ

Постановка краевой задачи. Пусть $G$ - область в $\mathbb{R}^{3}$, совпадающая вне большого шара с объединением непересекающихся полуцилиндров $\Pi_{+}^{q}=$ $\left\{\left(y^{q}, t^{q}\right): y^{q} \in \Omega^{q}, t^{q}>0\right\}$, где $q=1, \ldots, \mathscr{T}<\infty$ и $\Omega^{q}$ - ограниченная область в $\mathbb{R}^{2}$; граница $\partial G$ предполагается гладкой. В области $G$ рассматривается система Максвелла

с краевыми условиями

$$
\begin{aligned}
i \operatorname{rot} u^{2}(x)-k u^{1}(x) & =0, \\
-i \operatorname{div} u^{2}(x) & =0, \\
-i \operatorname{rot} u^{1}(x)-k u^{2}(x) & =0, \\
i \operatorname{div} u^{1}(x) & =0
\end{aligned}
$$

$$
\nu(x) \times u^{1}(x)=0, \quad\left\langle u^{2}(x), \nu(x)\right\rangle=0, \quad x \in \partial G,
$$

где $\langle\cdot, \cdot\rangle$ - скалярное произведение в $\mathbb{C}^{3}, \cdot \times \cdot-$ векторное произведение в $\mathbb{R}^{3}$, а $\nu$ - единичный вектор внешней нормали к $\partial G$. Положим $U=\left(u^{1}, u^{2}\right)$ и систему (1), (2) запишем в виде $\mathscr{M}(k) U=0$.

Собственные функции непрерывного спектра. Если для числа $k$ существует решение $U=\left(u^{1}, u^{2}\right)$ задачи $(1),(2)$ с оценкой $U(x)=O(|x|)$ при $|x| \rightarrow \infty$, не принадлежащее $L_{2}(G)$, то говорят, что $k$ - точка непрерывного спектра, а $U$ - отвечающая числу $k$ собственная функция непрерывного спектра. Известно [2], что непрерывный спектр задачи (1), (2) имеет вид $\left\{k \in \mathbb{R}:|k| \geqslant \mu_{\mathfrak{M}}^{1 / 2}\right\} \cup\{0\} ;$ здесь $\mu_{\mathfrak{M}}-$ наименьшее из положительных собственных чисел задач Неймана для оператора Лапласа $-\Delta$ в областях $\Omega_{q}$, $q=1, \ldots, \mathscr{T}$. Точка $k=0$ формально принадлежит непрерывному спектру, однако матрица рассеяния в этой точке не вводится, поэтому мы исключим ее из рассмотрения. Число $k$ называется собственным числом задачи (1), (2), если существует решение этой задачи, лежащее в $L_{2}(G)$; такое решение называется

* Исследование выполнено при поддержке грантов СПбГУ №11.38.666.2013 и РФФИ-1201-00247a. 
собственной функцией. Собственные числа не сгущаются на конечном расстоянии, а собственные функции экспоненциально затухают на бесконечности. Через $\operatorname{ker} \mathscr{M}(k)$ обозначим собственное пространство, натянутое на собственные функции, отвечающие собственному числу $k$. Пространство $\mathscr{E}(k)$, натянутое на собственные функции непрерывного спектра, содержит, в частности, собственное пространство $\operatorname{ker} \mathscr{M}(k)$. Кратностью $\varkappa(k)$ непрерывного спектра в точке $k$ назовем размерность факторпространства $\mathscr{E}(k) / \operatorname{ker} \mathscr{M}(k)$. Число $\varkappa(k)$ конечно для всех $k$, функция $k \rightarrow \varkappa(k)$ четная, кусочно постоянная и возрастающая с увеличением $|k|$; ее точки разрыва называются порогами. Положительные пороги образуют последовательность, возрастающую к бесконечности. На каждом интервале между двумя соседними порогами значение $\varkappa(k)$ остается постоянным. Далее мы рассматриваем $k$ на отрезке $\left[k^{\prime}, k^{\prime \prime}\right]$, свободном от порогов.

Матрица рассеяния. В пространстве $\mathscr{E}(k) / \operatorname{ker} \mathscr{M}(k)$ существует базис с представителями $\left\{Y_{1}^{+}, \ldots, Y_{\varkappa}^{+}\right\}$, такими, что

$$
Y_{j}^{+}(\cdot, k)=u_{j}^{+}(\cdot, k)+\sum_{l=1}^{\varkappa} s_{j l}(k) u_{l}^{-}(\cdot, k)+O\left(e^{-\delta|x|}\right), \quad j=1, \ldots, \varkappa,
$$

при больших $|x|$ и для всех $\delta<\delta_{0}$; здесь $u_{j}^{+}\left(u_{j}^{-}\right)$- «приходящие (уходящие) волны». При больших $|x|$ каждая из них удовлетворяет системе (1), (2), причем на множестве $\Pi_{+}^{q}$ для некоторого $q$ и больших $|x|$

$$
u_{j}^{ \pm}\left(y^{q}, t^{q} ; k\right)=\left|2 \lambda_{j}(k) k\right|^{-1 / 2} \exp \left(\mp i \lambda_{j}(k) t^{q}\right) \Phi_{j}^{\mp}\left(y^{q} ; k\right)
$$

и $u_{j}^{ \pm}(\cdot ; k)=0$ на $\Pi_{+}^{r}$ при $r \neq q$. Матрица $s(k)=\left\|s_{j l}(k)\right\|$ не зависит от выбора представителей в упомянутом базисе, унитарна и называется матрицей рассеяния.

Эллиптическое расширение системы Максвелла. Введем оператор $\{\mathscr{A}(D, k), \mathscr{B}\}$ эллиптической краевой задачи в области $G$,

$$
\begin{gathered}
\mathscr{A}(D, k) \mathscr{U}=\left(\begin{array}{c}
i \operatorname{rot} u^{2}+i \nabla a^{2}-k u^{1} \\
-i \operatorname{div} u^{2}-k a^{1} \\
-i \operatorname{rot} u^{1}-i \nabla a^{1}-k u^{2} \\
i \operatorname{div} u^{1}-k a^{2}
\end{array}\right), \quad x \in G, \\
\mathscr{B} \mathscr{U}=\left(-\left\langle u^{1}, \tau_{2}\right\rangle,\left\langle u^{1}, \tau_{1}\right\rangle,\left\langle u^{2}, \nu\right\rangle, a^{2}\right), \quad x \in \partial G,
\end{gathered}
$$

где $\mathscr{U}=\left(u^{1}, a^{1}, u^{2}, a^{2}\right), u^{j}-$ трехкомпонентные вектор-функции и $a^{j}-$ скалярные функции, $j=1,2$. Здесь $\tau_{1}, \tau_{2}, \nu$ - правая тройка ортонормированных векторов: $\nu$ - вектор внешней нормали к границе $\partial G$, а $\tau_{1}, \tau_{2}$ - касательные векторы. Оператор этой задачи самосопряжен относительно формулы Грина

$$
(\mathscr{A}(D, k) \mathscr{U}, \mathscr{V})_{G}+(\mathscr{B} \mathscr{U}, \mathscr{Q} \mathscr{V})_{\partial G}=(\mathscr{U}, \mathscr{A}(D, k) \mathscr{V})_{G}+(\mathscr{Q} \mathscr{U}, \mathscr{B} \mathscr{V})_{\partial G},
$$

справедливой для $\mathscr{U}, \mathscr{V} \in C_{c}^{\infty}\left(\bar{G} ; \mathbb{C}^{8}\right) ; \mathscr{Q} \mathscr{U}=-i\left(\left\langle u^{2}, \tau_{1}\right\rangle,\left\langle u^{2}, \tau_{2}\right\rangle, a^{1},-\left\langle u^{1}, \nu\right\rangle\right)$, $x \in \partial G$; наконец $(\cdot, \cdot)_{G}$ и $(\cdot, \cdot)$ дG - скалярные произведения в $L_{2}\left(G ; \mathbb{C}^{8}\right)$ и $L_{2}\left(\partial G ; \mathbb{C}^{4}\right)$ соответственно. (Далее в обозначениях пространств вектор-функций не указывается число компонент.) Отметим, что граничное условие $-\left\langle u^{1}, \tau_{2}\right\rangle=$ $\mathscr{G}^{1},\left\langle u^{1}, \tau_{1}\right\rangle=\mathscr{G}^{2}$ равносильно равенству $\nu \times u^{1}=\mathscr{G}^{1} \tau_{1}+\mathscr{G}^{2} \tau_{2}$ (см. (2)). 
Для задачи $\{\mathscr{A}(D, k), \mathscr{B}\} \mathscr{U}=0$ вводятся собственные функции непрерывного спектра, волны и матрица рассеяния [1]. На интервале $k \in\left[k^{\prime}, k^{\prime \prime}\right]$ кратность непрерывного спектра $\Upsilon(k)$ постоянна, матрица рассеяния $S(k)$ блочнодиагональная и один из ее блоков совпадает с $s(k)[2]$.

Вспомогательная задача в усеченной области. Положим $\Pi_{+}^{q, R}=\left\{\left(y^{q}, t^{q}\right)\right.$ $\left.\in \Pi_{+}^{q}: t^{q}>R\right\}, G^{R}=G \backslash \bigcup_{q=1}^{\mathscr{T}} \Pi_{+}^{q, R}$ при больших $R$. Тогда $\partial G^{R} \backslash \partial G=\Gamma^{R}=$ $\bigcup_{q=1}^{\mathscr{T}} \Gamma^{q, R}$, где $\Gamma^{q, R}=\left\{\left(y^{q}, t^{q}\right) \in \Pi_{+}^{q}: t^{q}=R\right\}$. Введем эллиптическую краевую задачу

$$
\begin{aligned}
\mathscr{A}(D, k) \mathscr{U}(x) & =\mathscr{F}(x), & & x \in G^{R}, \\
\mathscr{B}(x) \mathscr{U}(x) & =\mathscr{G}(x), & & x \in \partial G^{R} \backslash \Gamma^{R}, \\
(\mathscr{B}(x)+i \mathscr{Q}(x)) \mathscr{U}(x) & =\mathscr{H}(x), & & x \in \Gamma^{R} .
\end{aligned}
$$

Граница $\partial G^{R}$ содержит ребра $\partial \Gamma^{q, R} ;$ пусть $\partial \Gamma^{R}=\bigcup_{q=1}^{\mathscr{T}} \partial \Gamma^{q, R}$. Пусть, кроме того, $r$ - гладкая положительная на $\overline{G^{R}} \backslash \partial \Gamma^{R}$ функция, совпадающая вблизи $\partial \Gamma^{R}$ с расстоянием $\operatorname{dist}\left(x, \partial \Gamma^{R}\right)$. Через $H_{\beta}^{l}\left(G^{R}\right), l=0,1$, обозначим замыкание множества $C_{c}^{\infty}\left(\overline{G^{R}} \backslash \partial \Gamma^{R}\right)$ по норме

$$
\left\|u ; H_{\beta}^{l}\left(G^{R}\right)\right\|=\left(\sum_{|\alpha| \leqslant l} \int_{G^{R}} r(x)^{2(\beta-l+|\alpha|)}\left|D_{x}^{\alpha} u(x)\right|^{2} d x\right)^{1 / 2},
$$

а через $H_{\beta}^{1 / 2}\left(\Gamma^{R}\right)$ и $H_{\beta}^{1 / 2}\left(\partial G^{R} \backslash \Gamma^{R}\right)$ пространства следов функций из $H_{\beta}^{1}\left(G^{R}\right)$ на $\Gamma^{R}$ и $\partial G^{R} \backslash \Gamma^{R}$.

Теорема 1. Существует число $\varepsilon>0$, такое, что при $\beta \in(1 / 2-\varepsilon, 1 / 2+\varepsilon) u$

$$
\{\mathscr{F}, \mathscr{G}, \mathscr{H}\} \in H_{\beta}^{0}\left(G^{R}\right) \times H_{\beta}^{1 / 2}\left(\partial G^{R} \backslash \Gamma^{R}\right) \times H_{\beta}^{1 / 2}\left(\Gamma^{R}\right),
$$

задача (4) имеет единственное решение $\mathscr{U} \in H_{\beta}^{1}\left(G^{R}\right)$. Если $\mathscr{F}=0, \mathscr{G}=0$ u $\beta \leqslant 1 / 2$, то для решения $\mathscr{U}$ выполняется неравенство $\left\|\mathscr{Q} \mathscr{U} ; L_{2}\left(\Gamma^{R}\right)\right\| \leqslant$ $\left\|\mathscr{H} ; L_{2}\left(\Gamma^{R}\right)\right\|$.

Функционал и теорема о сходимости. Пусть $\mathscr{U}_{j}^{ \pm}, j=1, \ldots, \Upsilon,-$ волны задачи $\{\mathscr{A}(D, k), \mathscr{B}\} \mathscr{U}=0$. Обозначим через $X_{l}^{R}(\cdot, k ; a)$ решение задачи $(4) \mathrm{c}$ правой частью

$$
\mathscr{F}=0, \quad \mathscr{G}=0, \quad \mathscr{H}=(\mathscr{B}+i \mathscr{Q})\left(\mathscr{U}_{l}^{+}(\cdot, k)+\sum_{j=1}^{\Upsilon} a_{j} \mathscr{U}_{j}^{-}(\cdot, k)\right),
$$

где $a=\left(a_{1}, \ldots, a_{\Upsilon}\right)-$ произвольный вектор из $\mathbb{C}^{\Upsilon}$. Введем функционал

$$
\mathbb{C}^{\Upsilon} \ni a \mapsto J_{l}^{R}(a ; k)=\left\|\mathscr{Q}\left(X_{l}^{R}(\cdot, k ; a)-\mathscr{U}_{l}^{+}(\cdot, k)-\sum_{j=1}^{\Upsilon} a_{j} \mathscr{U}_{j}^{-}(\cdot, k)\right) ; L_{2}\left(\Gamma^{R}\right)\right\|^{2} .
$$

Пусть $Z_{j, R}^{ \pm}$- решение задачи (4) при $\mathscr{F}=0, \mathscr{G}=0, \mathscr{H}=(\mathscr{B}+i \mathscr{Q}) \mathscr{U}_{j}^{ \pm}(\cdot, k)$. Имеем $X_{l}^{R}=Z_{l, R}^{+}+\sum a_{j} Z_{j, R}^{-}$. Теперь функционал принимает вид

$$
J_{l}^{R}(a ; k)=\left\langle a E^{R}, a\right\rangle+2 \operatorname{Re}\left\langle F_{l}^{R}, a\right\rangle+G_{l}^{R},
$$


где $E^{R}, F^{R}$ - матрицы размера $\Upsilon \times \Upsilon$ с элементами $E_{i j}^{R}=\left(\mathscr{Q}\left(Z_{i, R}^{-}-\mathscr{U}_{i}^{-}\right), \mathscr{Q}\left(Z_{j, R}^{-}-\mathscr{U}_{j}^{-}\right)\right)_{\Gamma^{R}}, \quad F_{i j}^{R}=\left(\mathscr{Q}\left(Z_{i, R}^{+}-\mathscr{U}_{i}^{+}\right), \mathscr{Q}\left(Z_{j, R}^{-}-\mathscr{U}_{j}^{-}\right)\right)_{\Gamma^{R}}$, вектор $F_{l}^{R}$ - строка с номером $l$ матрицы $F^{R}$, а $G_{l}^{R}=\left\|\mathscr{Q}\left(Z_{l, R}^{+}-\mathscr{U}_{l}^{+}\right)\right\|_{\Gamma^{R}}^{2}$.

В качестве приближения для строки $\left(S_{l 1}, \ldots, S_{l \Upsilon}\right)$ матрицы рассеяния $S(k)$ выберем минимизатор $a^{0}(R, k)=\left(a_{1}^{0}(R, k), \ldots, a_{\Upsilon}^{0}(R, k)\right)$ функционала $(5)$. Из (6) следует, что минимизатор $a^{0}(R, k)$ является решением системы

$$
a^{0}(R, k) E^{R}+F_{l}^{R}=0,
$$

а приближение $S^{R}$ для матрицы рассеяния, состоящее из таких строк, удовлетворяет равенству $S^{R} E^{R}+F^{R}=0$.

Теорема 2. Пусть отрезок $\left[k^{\prime}, k^{\prime \prime}\right]$ непрерьвного спектра задачи (1), (2) не содержит порогов. Тогда для всех $k \in\left[k^{\prime}, k^{\prime \prime}\right] \quad u \quad R>R_{0}$, где $R_{0}$ - достаточно большое число, существует единственный минимизатор $a^{0}(R, k)$ функиионала (5), причем

$$
\left|a_{j}^{0}(R, k)-S_{l j}(k)\right| \leqslant C e^{-\delta R}, \quad j=1, \ldots, \Upsilon
$$

здесь $\delta<\delta_{0}-$ то же число, что и в (3), а постоянная $C$ не зависит ни от $k$, ни от $R$.

Так как матрица $S(k)$ блочно-диагональная, приближение для матрицы $s(k)$ дается блоком матрицы $S^{R}$. Метод допускает модификацию для вычисления матрицы рассеяния в окрестности порогов; для оператора Гельмгольца такая модификация описана в [4].

\section{ЛитЕРАТУРА}

[1] С. А. Назаров, Б. А. Пламеневский, Эллиптические задачи в областях с кусочно гладкой гранищей, Наука, М., 1991. [2] Б. А. Пламеневский, А. С. Порецкий, Алгебра и анализ, 25:1 (2013), 94-155. [3] Б. А. Пламеневский, О. В. Сарафанов, Функц. анализ и его прил., 48:1 (2014), 61-72. [4] Б. А. Пламеневский, А. С. Порецкий, О. В. Сарафанов, Алгебра и анализ, 26:1 (2014), 128-164.

Санкт-Петербургский государственный университет, физический факультет

Поступила в редакцию e-mail: boris.plamen@gmail.com 10 октября 2014 г.

Санкт-Петербургский государственный университет, физический факультет

Faculty of Information Technology, University of Jyväskylä

e-mail: poras1990@list.ru

Санкт-Петербургский государственный университет,

физический факультет

e-mail: saraf@math.nw.ru

Заведующая редакцией и научный редактор Г. М. Цукерман

Подписано к печати 22.01.2015. Дата выхода в свет 20.02.2015. Формат 70×100/16 Печать цифровая. Усл. печ. л. 7,8. Усл. кр.-отт. 1,7 тыс. Бум. л. 3,0 Уч.-изд. л. 8,0. Тираж 158 экз. Заказ 925. 